\title{
Parameter Exploration for Response Time Reduction in Accelerometer- based Activity Recognition
}

Minoru Yoshizawa

Toyohashi University Of

Technology

minoru@usl.cs.tut.sc.jp

\section{Wataru Takasaki}

Taihei Kogyo, Co., Ltd.

Ren Ohmura

Toyohashi University Of

Technology

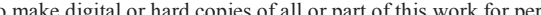
ranted without fee provided that copies are not made or distributed for profit or commercial advanta and that copies bear this notice and the full citation on the first page. Copyrights for components
work owned by others than ACM must be honored. Abstracting with credit is permitted. To copy otherwise, or republish, to post on servers or to redistribute to lists, requires prior specific permissio and/or a fee. Request permissions from Permissions@acm.or

UbiComp '13, September 08 - 12 2013, Zurich, Switzerland

http://dx.doi.org/10.1145/2494091.2495986.

\begin{abstract}
In activity recognition techniques, existing wearable sensors have a problem performing the recognition process. Because existing wearable sensors perform recognition process by dividing sensor data into partial sequences, there is lag between the changes in action and the output of the recognition result. Therefore, we focused on the point activities change and have proposed a method to reduce the response time of the activity recognition technique. However, parameters such as window size immediately after the activity changing point and the activity changing point detection in the proposed method have not been studied well. Thus, in this paper, we conducted experiments using the HASC Corpus, which contains large-scale data of human activity. We report results of examining various parameters in the proposed method and features of the proposed method revealed by comparison with a conventional method. To give a concrete example, for IIR band-pass filter bank to be used for activity changing point detection, we clarified the frequency and the appropriate number of filters. In addition, we clarified the relationship between

identification accuracy and the size of a special window that is set after activity changing point detection. The proposed method reduced the response time to the $2035 \mathrm{~ms}$ on average from $2773 \mathrm{~ms}$, the of average of the conventional method. In addition, the proposed method can reduce the amount of calculation, achieve both high recognition accuracy and short response time,
\end{abstract}


and output the recognition results in consistent times to reduce the jitter of response time.

\section{Author Keywords}

Activity recognition; wearable sensor; response time; including recognition accuracy.

\section{ACM Classification Keywords}

H.5.m. Information interfaces and presentation (e.g., $\mathrm{HCI})$ :

Miscellaneous.

\section{General Terms}

Algorithm; experimentation; performance.

\section{Introduction}

Activity recognition is a key technology for implementing a context-aware system. It is expected to be utilized in the field of life-logs, operation of a device by gesture, and work analysis. With the size and weight of the sensor devices becoming smaller and lighter in recent years, the activity recognition based on wearable sensors, such as an acceleration sensor mounted on the body, have been widely studied.

The existing activity recognition technique first divides sensor data into partial sequences that have several time widths by using a sliding window method. Then, it performs the identification process based on the machine learning the subsequence by using the partial sequences as an instance. There is a problem that existing activity recognition technique lacks real-time property because it performs the division process in such partial sequences. It cannot recognize a real action immediately. Therefore, in many cases, the activity recognition is difficult to apply to the system requiring immediate responses such as reminders or device control based on recognized behavior.

There have been few studies on more quickly obtaining recognition results from the input of the sensor data.

Mr. Fujimoto proposed two motion recognition method for dance performances[3]. Specifically, One is adaptively decisions recognition of the size of the window. The other is movement recognition by twostep method to achieve higher accuracy in high-speed recognition. Although this research was intended to achieve high-speed response of activity recognition, this method is difficult to adapt to general daily behavior because it is for obtaining a high speed response by optimizing the recognition timing on the basis of the timing of BGM.

Therefore, we focus on the activities that change at arbitrary times and take particular note of the point at which activities change. Additionally, we propose a method for reducing the time between an activity actually changing and the recognition result of the activity changing being outputted (in this research, we just call this "response time") [12]. We detect an "activity changing point of activity" from sensor data directly, and we are planning to reduce the response time by operating a recognition algorithm using a window shorter than usual. The activity changing point is detected by auto segmentation technique which is based on the STM(Spectral Transition Measure). Of the experimental evaluation results revealed that the proposed method reduced the average response time by about 1 second. However, as we discuss later, although the proposed method needed to set various parameters for activity changing point detection, window size, etc., they were decided intuitively and were not considered thoroughly. 


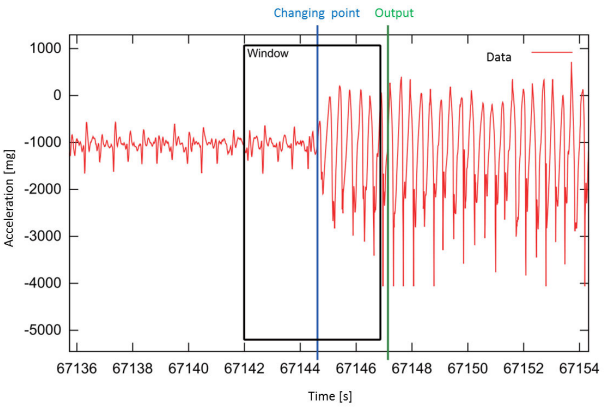

Figure 1. Window overlapping

activity changing point of activity

Moreover, the data used in the evaluation are based on the small-scale data acquired from two subjects and so were unreliable.

In this paper, we review the technique proposed by Moreover, the characteristic of the proposed method is clarified by comparison with the conventional method. For evaluation data, we used the data of about 41 people and 50 datasets from the HASC2012 corpus, which is the data acquired by HASC, which collects large-scale activity data.

\section{PROPOSED METHOD}

Cause of Recognition Delay in Existing Method As mentioned above, a behavioral-recognition method is used in the sliding window method. The sliding window divides sensor data into partial series by applying a window function to sensor data. In the classification processing, this subsequence is executed as one instance and outputs a result. Next, window function is displaced to the later time. Then the window function is processed in a similar fashion. The sliding window method works to distinguish these processes

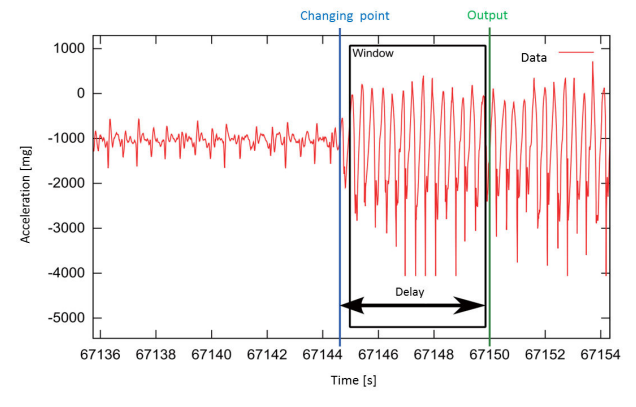

Figure 2. Configration window after changing activity

continually, repeatedly, and sequentially. Therefore, the width of the window function and window shift in addition to the form of the window function becomes a parameter of the sliding window.

Positions of the windows are usually decided when an action starts with the sliding window. For example, the window steps over the activity changing point and may be set even if an action changed, such as in figure 1 , at a certain point in time. The features calculated from such a window become a calculated subsequence which before and after the activity changing point were mixed, and the recognition rate of subsequence decreases. By chance, when a window is set right after an action changes, the features to be provided from a window are calculated by the sensor data only for activities after the change. This change is expected to be accurately recognized (figure 2 ). However, since recognition is performed after the sensor data for window size are gathered, the result of recognition is obtained after the time for window size since the activity changed. This recognition is much slower than the present activity. In this way, precision of the recognition lowers when an activity changes with the normal sliding window, and the recognition is much slower than the present activity. 


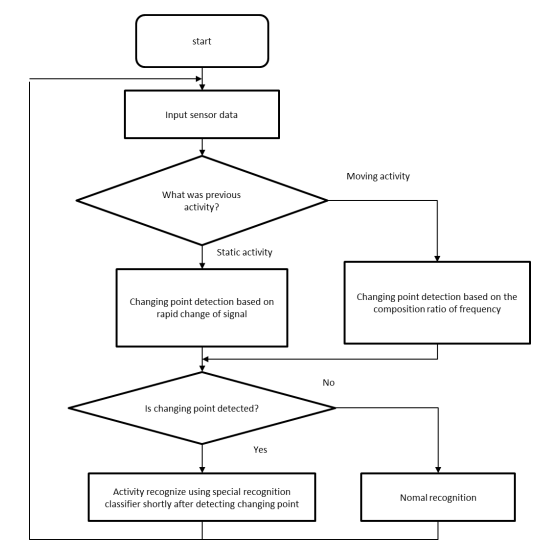

Figure 3. Flow chart of proposed method
Overview of Proposed Method

Therefore, we proposed a technique for shortening the response time of recognizing the activity changing point of activities [12]. As Figure 3 shows, the proposed method performs recognition using the usual sliding window while the recognized activity does not change. On the other hand, we set a window just after an activity changing point when we detect that an action has changed. At this time, as figure 4 shows, we plan the exact recognition and shorting of response time by using the specialized recognition equipment for the activity changing point in the shorter-than-usual window width. In next subsection, we state the surveyed parameters of each procedure in this research and explain each procedure.

\section{Activity changing point Detection}

Activity changing point detection was prepared with reference to Murao et al.'s proposed method [11]. We distinguish between static activity and moving activity. Static activities have potential to change another activity from "stand" and "sit" Similarly, moving activities have potential to change another activity from "walk" and "run". In case of the transit from static activity, we use tube which is proposed by Murao et al's. In case of the transit from moving activity, we use STM which is reference to Omura et al.'s proposed method [7]. Activity is determined to be either "static activity" or "moving activity" on the basis of the result of activity recognition. Each method is described in later sections.

Activity changing point Detection from Static Activity Activity changing point from static activity is detected by detecting rapid change in sensor data by using a $\epsilon$ tube. As Figure 5 and Eq. 1 show, if the difference between the moving average of $\bar{x}$ the sensor data for the past $\mathrm{T}$ seconds and the current sensor data is more than threshold $\epsilon$, this point is used as the activity

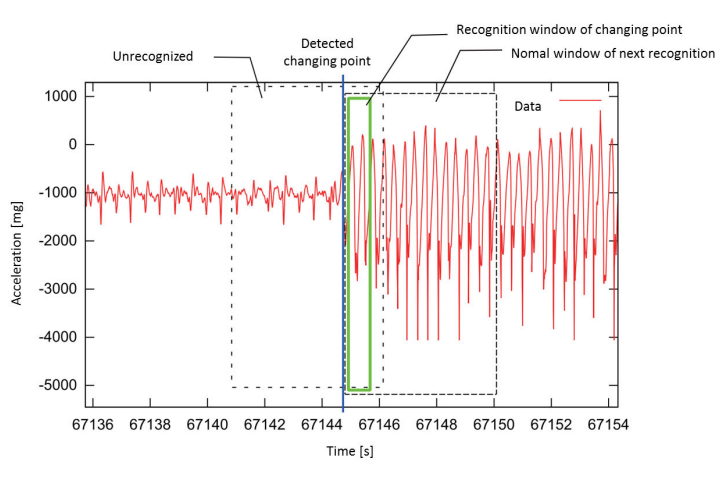

Figure 4. Setting of recognize window when activity changing point is detected

changing point. The activity recognition system usually uses tri-axial ( $x, y$, and $z$ axes) acceleration sensors.

Also, these sensors are usually made of multiple combined sensors. In the proposed method, completeness (recall) has priority over accuracy (precision), and if a change has been detected at a given time, this is used as the activity changing point.

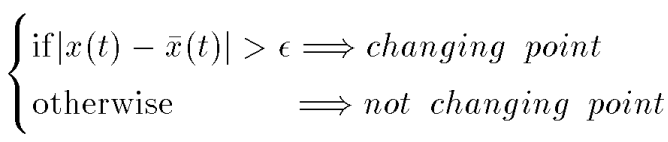

Activity changing point Detection from Moving Activity Activity changing points from transit moving activity to another activity is detected by using Omura et al.'s proposed method [7] extended for real time. Omura et al.'s proposed method is based on research on automatic segmentation of speech wave by Furui and calculates the spectrum transition rate $G(t)$, which is defined by the following equation. 


$$
\begin{gathered}
G(t)=\sum_{i=1}^{p} \frac{a^{2}}{p} \\
a_{i}(t)=\frac{\sum_{n=-M}^{M} C_{i}(t+n) n}{n^{2}}
\end{gathered}
$$

We continuously calculated this spectrum transition rate $G(t)$, and point in time when a peak appeared in $G(t)$ was used as the activity changing point. Here, $C_{i}(n)$ is a frequency-domain feature of $i$-dimensional coefficient in the $p$-dimensions at the time of $\mathrm{n}$ (the nth window), and Omura et al.'s proposed method is used in Cepstral features (Furui et al. proposed method is used in Mel-frequency cepstrum coefficients). M shows the number of the anteroposterior windows of the target window. However, if spectrum transition defined by the above equation is used as is, it become delayed due to detection being retroactively required. To begin with, Eq. 3 shows that we need to future data which is over after the activity changing point data. This introduces the possibility of defining real time sensor data as future data which is over after the activity changing point data. Also, Fast Fourier Transform (FFT) is generally used to obtain frequency components from time-series signal and calculate

$C_{i}(n)$ (Cepstral features). Because FFT is processing for a certain section, the processing is delayed until data are gathered.

Thus, the proposed method uses IIR band-pass filter to obtain frequency components from time-series sensor data as fast as possible and to consider whether sum of variation exceeds a certain value for each component in an activity changing point. In particular, a signal obtained from sensor data is first divided into signals

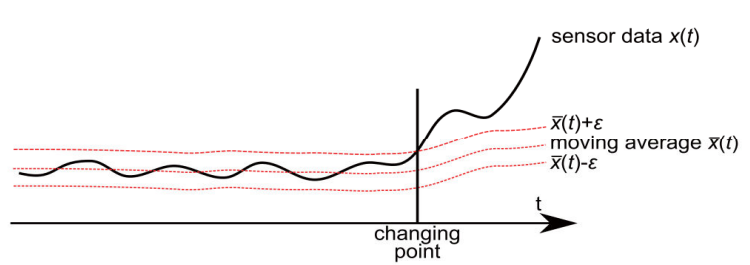

Figure 5. Activity changing point detection using $\epsilon$ tube

made of the different frequency band by a pass filter bank made of IIR band pass filters. Then, let signal intensity be the peak absolute value for nearest $y_{i}(t)$ (Eq. 4). Furthermore, we calculate moving average $\bar{p}_{i}(t)$ of signal intensity of each frequency for the past $T$ seconds to use the moving average filter. We calculate a composition ratio $c r_{i}(t)$ of the current power signal of the each frequency and a composition ratio crma $(t)$ of the power signal between T seconds of each frequency of the moving average (Eqs. 5 and 6 ). Then, we calculate the sum of the square of these composition ratios, and if this value exceeds the threshold, it is used as the activity changing point (Eq. 7).

As is the case in activity changing point detection from static activity, if an activity changing point is detected in any sensor data at a given time, it is used as an activity changing point.

$$
p_{i}(t)=\left\{\begin{array}{cc}
y_{i}(t-1) & \text { if }\left|y_{i}(t-1)\right|>\left|y_{i}(t-2)\right| \\
& \text { and }\left|y_{i}(t-1)\right|>\left|y_{i}(t)\right| \\
p_{i}(t-1) & \text { otherwise }
\end{array}\right.
$$




$$
\begin{gathered}
c r_{i}(t)=\frac{p_{i}(t)}{\sum_{k=1}^{n} p_{k}(t)} \\
\operatorname{crma}_{i}(t)=\frac{\bar{p}_{i}(t)}{\sum_{k=1}^{n} \bar{p}_{k}(t)} \\
\left\{\begin{aligned}
\sum_{k=1}^{n}\left(c r_{k}(t)-c r m a_{k}(t)\right)^{2}>\text { threshold } \\
\Rightarrow \text { changing point }
\end{aligned}\right. \\
\text { otherwise not changing point }
\end{gathered}
$$

Here, the parameters we should adjust are the different frequency band of $y_{i}(t)$, its number, and its threshold In addition, the frequency band and its number are the passing frequency of IIR band pass filter and number of filters, respectively.

\section{RECOGNITION}

While activity changing point is not detected,

recognition is performed by setting a recognition window by a normal sliding window. Meanwhile, if activity changing point is detected, the specialized recognition equipment is required for recognizing the activity changing point of the shorter-than-usual window width. At that time, the normal sliding window is stopped temporarily.

We use the detected activity changing point as the starting point for high-speed response in activity recognition and make the recognition window smaller than usual. The recognition using this window requires the use of specialized recognition equipment that gathers only data just after activity changing point using small window size. In addition, when we set the recognition window of the activity changing point, we similarly resume recognition of the normal sliding window using the activity changing point as the starting point.

Here the width of the window for the activity changing point is the main parameter we should adjust.

\section{EXPERIMENT}

In the proposed technique so far, the experiments were carried out and compared with conventional methods after the parameters were examined. Regulated parameters were :

- The frequency and number of IIR band pass filters for activity changing point detection in an active condition.

- The range of a small window set up after changing point detection.

Besides these parameters, $\epsilon$ of $\epsilon$-tube essentially stated in a previous subsection (Activity changing point Detection from Static Activity) also is contained in the range of verification. However, as shown in Fig. 6, micro vibration of acceleration seen as a noise remained less than about $0.1 \mathrm{G}$ in many cases. When the value of $\epsilon$ is too small, it is so sensitive that it responds even very minor activity, so $\epsilon$ was locked at 0.2 , twice $0.1 \mathrm{G}$, and the other parameter was adjusted preferentially.

In the following subsection, the experiment environment is described.

\section{Experiment Environment}

The Net Frame work was used to mount a proposed technique and evaluation environment. SVM (SMO) of Weka [9], which is an open source data mining tool, is used for the discrimination algorithm in activity

recognition. In the feature amount, average, standard 


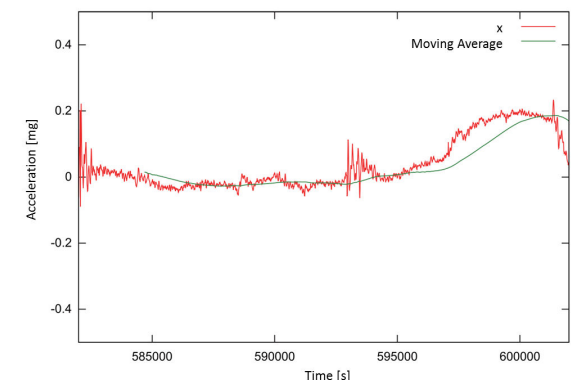

Figure 6. Sensor data of static state

deviation, and coefficient of correlation between each axis of the sensor are used.

In this research, HASC Corpus (2012 edition) is referred to as experimental data. The HASC Corpus is the annotation data, which are gleaned from about 250 subjects collected by HASC [4] (Human Activity Sensing Consortium. The organization's purpose is "Formulation of a large scale database by wearable sensor to understand the human behavior."). Classifications of the sensor device to use, an installation place, the sampling frequency, and so on are not appointed varied. Additionally, only one sensor acquires data in many cases. There are six kinds of intended activities: "motionlessness (erectness)", "walking", "jogging", "skip", "climbing up stairs", and "climbing down stairs".

At a sampling frequency of $100 \mathrm{~Hz}, 50$ datasets were used for an experiment involving 41 people using acceleration data included in HASC2012 corpus, which was the result of the HASC Challenge. Furthermore, because "motionlessness" is a calm condition, a method for activity changing point detection was divided since the other five kinds condition are active activity.

\begin{tabular}{|r|r|r|r|}
\hline coefficient as feature value & precision & Recall & F measure \\
\hline $1,2,3$ & 53.5 & 33.1 & 40.9 \\
\hline $4,5,6$ & 42.1 & 27.4 & 33.2 \\
\hline $7,8,9$ & 33.2 & 27.1 & 29.8 \\
\hline $1,4,7$ & 47.9 & 29.7 & 36.7 \\
\hline $2,5,8$ & 33.7 & 24.7 & 28.5 \\
\hline $3,6,9$ & 34.9 & 23.0 & 27.7 \\
\hline $1,2,3,4,5$ & 52.7 & 34.9 & 42.0 \\
\hline $1,3,5,7,9$ & 53.1 & 34.5 & 41.8 \\
\hline $2,4,6,8,10$ & 36.7 & 26.1 & 30.5 \\
\hline $6,7,8,9,10$ & 37.5 & 32.5 & 34.8 \\
\hline
\end{tabular}

Table 1. Segmentation accuracy by STM

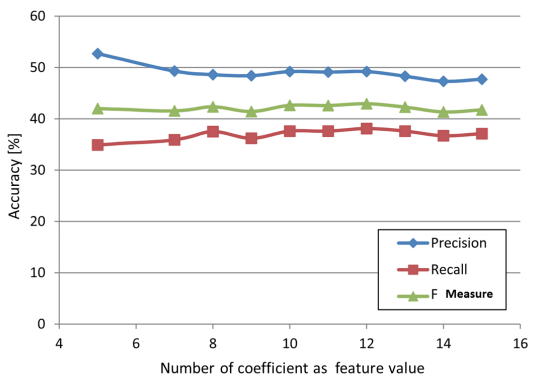

Figure 7. Segmentation accuracy by STM, which is used as aconsecutive feature.

The Parameter of IIR Filter Bank

The experiments were conducted to determine each passing frequency and filter bank used for detecting activity changing points in active behavior. As a basic consideration, at first, an activity changing point detection method was implemented by peak detection of spectrum degree of succession[7], and the sequence data of HASC Corpus were applied. The previous or next number of the intended windows is put at 10 , and 


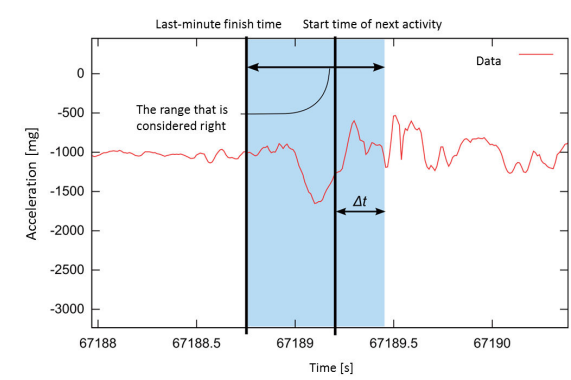

Figure 8. Effective range of activity changing point detection. The activity changing point detected in the section from the finish time of the action before being specified by labeling start time $\Delta \mathrm{t}$ second of the next action, this is regarded as "The activity changing point was detected correctly".

\begin{tabular}{|r|r|r|r|}
\hline number of coefficient & Precision & Recall & F measure \\
\hline 5 & 52.7 & 34.9 & 42.0 \\
\hline 7 & 49.3 & 35.9 & 41.5 \\
\hline 8 & 48.6 & 37.5 & 42.3 \\
\hline 9 & 48.4 & 36.2 & 41.4 \\
\hline 10 & 49.2 & 37.6 & 42.6 \\
\hline 11 & 49.1 & 37.6 & 42.6 \\
\hline 12 & 49.2 & 38.1 & 42.9 \\
\hline 13 & 48.3 & 37.6 & 42.3 \\
\hline 14 & 47.3 & 36.7 & 41.3 \\
\hline 15 & 47.7 & 37.1 & 41.7 \\
\hline
\end{tabular}

Table 2. Segmentation accuracy by STM which is used a consecutive feature.

examination was conducted to see whether the Cepstral feature was varied as a feature amount and which signal of frequency is important for segmentation. Then, window size was given as 2560 ms and the Cepstral coefficient was calculated.

The accuracy of segmentation (abstract) for the case of coefficient varied as feature amount is shown in table 1. As a result, it can indicate that the case containing the primary coefficient is more sensitive than the case not containing the primary coefficient as a tendency. In case of each number of characters are successive feature values in order from primary coefficient, segmentation outperforms intermittently case. In the second place, the accuracy of segmentation in the case in which the number was varied is shown in table 2 and Fig. 7.

For $F$ value and recall ratio, the segmentation was highest when Cepstral feature was characterized in succession from first to twelfth. As mentioned above, it is appropriate to apply a recall ratio up to twelve because the activity changing point detection should attach great importance to a recall.

\begin{tabular}{r|cc|}
\hline \multicolumn{1}{|c|}{ No. } & passing frequency $[\mathrm{Hz}]$ & passing wavelength $[\mathrm{ms}]$ \\
\hline 1 & 0.391 & 2558 \\
\hline 2 & 0.781 & 1280 \\
\hline 3 & 1.172 & 853 \\
\hline 4 & 1.563 & 640 \\
\hline 5 & 1.953 & 512 \\
\hline 6 & 2.344 & 427 \\
\hline 7 & 2.734 & 365 \\
\hline 8 & 3.125 & 320 \\
\hline 9 & 3.516 & 284 \\
\hline 10 & 3.906 & 256 \\
\hline 11 & 4.297 & 232 \\
\hline 12 & 4.688 & 213 \\
\hline
\end{tabular}

Table 3. Passing frequency of filter bank

According to the above results, 12 filters of a filter bank are used to detect the activity changing point from active behavior, and passing frequency of each IIR filter is set as indicated in table 3 . The IIR degree of the filter was put in a minimal secondary filter to speed up the response time.

Evaluation of Delay of Activity changing point Detection Next, the delay of activity changing point detection was evaluated using the frequency and the number of filters specified above. We evaluated the relation of the delay and accuracy. In the evaluation, as shown in Fig. 8 when the activity changing point detected in the section from the finish time of the action before being specified by labeling start time $\Delta \mathrm{t}$ second of the next action, this is regarded as "The activity changing point was detected correctly". Conversely, suppose that "misdetection" is when the activity changing point detected in this range does not exist. To check what delay arises, the value of $\Delta \mathrm{t}$ is increased by $100 \mathrm{~ms}$ from $100 \mathrm{~ms}$ to $3000 \mathrm{~ms}$, and the accuracy and the recall are asked for at each $\Delta \mathrm{t}$.

Fig. 9 shows the results. The accuracy and recall 


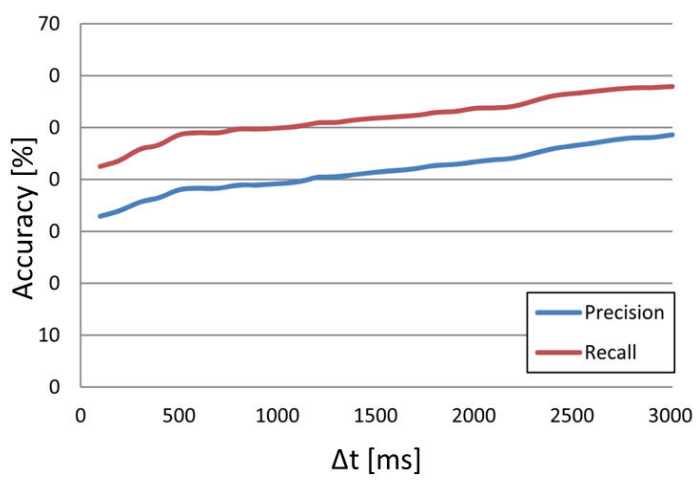

Figure 9. Accuracy of activity changing point detection

\begin{tabular}{r|c|c|}
\hline WindowSize[ms] & Precision[\%] & Recall[\%] \\
\hline 160 & 40.5 & 34.8 \\
\hline 320 & 45.5 & 39.9 \\
\hline 640 & 49.7 & 47.0 \\
\hline 1280 & 60.8 & 61.5 \\
\hline 2560 & 67.3 & 69.9 \\
\hline
\end{tabular}

Table 4. Window size and recognition accuracy of activity changing point

increase linearly as time passes. Specifically, accuracy tend to increase: even through $\triangle \mathrm{t}$ attained the recall of about $50 \%$ in the stage for $500 \mathrm{~ms}$ and set $\Delta \mathrm{t}$ to $3000 \mathrm{~ms}$, it did not reach $60 \%$.

Evaluation of Window Size at the Time of Activity changing point

To examine the suitable size for the window specified when an activity changing point is detected, we experimented by assuming that the activity changing

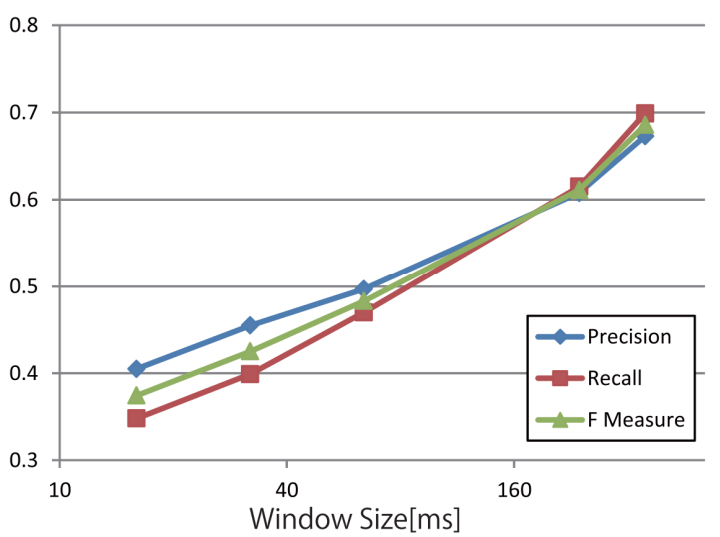

Figure 10. Window size (logarithm) and recognition accuracy at activity changing points

point was detected correctly. Specifically in an annotation label, a recognition window is specified immediately after the starting point of a certain action. The window size after an activity changing point was set to $160 \mathrm{~ms}, 320 \mathrm{~ms}, 640 \mathrm{~ms}, 1280 \mathrm{~ms}$, and $2560 \mathrm{~ms}$.

Table 4 and Fig. 10 show the accuracy of the recognition in each window size obtained in this experiment. In addition, the time-axis of Fig. 10 is presented as the logarithmic scale. As expected, if window is enlarged, the recognition rate increases, and conversely, if window is shrunk, accuracy and recall decrease.

The degree of the increase or decrease is linear mostly to the logarithm of time. Windows do not generally need to be large, it is regarded as delay until a recognition result is obtained. For this reason, one value as an "optimal" parameter is difficult to determine, and values need to be appropriately determined in accordance with the response time needed. 
Comparison with Conventional Technique To evaluate overall performance (recognition accuracy and response time), recognition was performed through a whole sequence data about the conventional technique (the method continues recognition continuously using fixed window width and shift width) and the proposed technique. At this time, the conventional technique changed window size to 320 to 2560 , and we experimented by changing shift width from every $10 \mathrm{~ms}$ (this is equivalent to a sample because a sampling rate is $100 \mathrm{~Hz}$ ) to window width. The sliding window was fixed to $2560 \mathrm{~ms}$ window width and $1280 \mathrm{~ms}$ shift width when activity changing point detection of the proposed technique is not performed. The frequency and the number of IIR band pass filters in activity changing point detection are determined by before section (Section name is "The Parameter of IIR Filter Bank"). We set window size after an activity changing point as $320 \mathrm{~ms}$.

Table 5 shows recognition accuracy and response time in the conventional technique and the proposed

technique. In addition, Failure Rate is the rate at which the right recognition result was not once obtained after an action changed before changing to another action.

Recognition accuracy of the proposed technique became almost equivalent to that of the conventional technique of the same window size $(2560 \mathrm{~ms})$ and shift width $(1280 \mathrm{~ms})$. Moreover, recognition accuracy of the conventional technique decreases if the window is small. On the other hand, shift width barely influences recognition accuracy. According to a comparison of the proposed technique and the conventional technique, response time can be shortened by applying the proposed technique from an average of $2773 \mathrm{~ms}$ to an average of $2035 \mathrm{~ms}$ if the window size and shift width are the same (window size $2560 \mathrm{~ms}$, shift width

$1280 \mathrm{~ms})$. However, when the proposed technique is not applied, the conventional technique can shorten response time by making window size and shift width small. In particular, in accordance with window shift's fluctuates, the possibility that a window will be set exactly after the activity changing point of action (or the window will try to include many feature quantities of action after a change if the activity changing point is straddled) will become high if shift width is short.

On the other hand, the standard deviation of the response time has little correlation with either window size and shift width. Moreover, while in all cases response time of conventional techniques is $3000 \mathrm{~ms}$ from about $2000 \mathrm{~ms}$, the response time of the proposed technique is $1000 \mathrm{~ms}$. Therefore, in all cases the response time of the proposed technique is more stable than that of the conventional technique.

Consideration

Although the response time of the action recognition in the activity changing point of action was shortened by $700 \mathrm{~ms}$ from $2773 \mathrm{~ms}$ to $2035 \mathrm{~ms}$ on average by using the proposed technique, by shortening window size and shift width, early response time can be obtained also with the conventional technique. However, when window size is shortened, overall recognition accuracy decreases. Moreover, when shift width is shortened, the frequency of discernment processing will rise and will increase the load of a computer. Although the

quantitative evaluation is not performed, in case of the obtained almost the same accuracy and average response time similar both of proposed methods, window width is $2560 \mathrm{~ms}$ and window shift is $160 \mathrm{~ms}$. However this case needs approximately eight times computational because of the processing of activity changing point detection needs high computational times. We may, therefore, reasonably conclude that the proposed technique can shorten response time and increase recognition accuracy, thus reducing computational complexity.

Moreover, the standard deviation of response time shows a very interesting result. Although, for the 


\begin{tabular}{|c|c|c|c|c|c|}
\hline Conventional method & & Recognize & accuracy & Respon & e time \\
\hline $\begin{array}{l}\text { WindowSize[ms] } \\
320\end{array}$ & $\begin{array}{l}\text { WindowShift }[\mathrm{ms}] \\
\end{array}$ & Precision[\%] & Recall[\%] & Average[ms] & $\frac{\operatorname{StdDev}[\mathrm{ms}]}{1922}$ \\
\hline $\begin{array}{l}320 \\
320\end{array}$ & $\begin{array}{r}10 \\
160\end{array}$ & $\begin{array}{l}57.6 \\
57.5\end{array}$ & $\begin{array}{l}50.0 \\
58.2\end{array}$ & $\begin{array}{c}712 \\
1013\end{array}$ & $\begin{array}{l}1922 \\
2130\end{array}$ \\
\hline 320 & 320 & 57.6 & 58.3 & 1205 & 2333 \\
\hline 640 & 10 & 62.9 & 64.7 & 860 & 2109 \\
\hline 640 & 160 & 62.9 & 64.7 & 1003 & 2178 \\
\hline 640 & 320 & 63.0 & 64.8 & 1303 & 2955 \\
\hline 640 & 640 & 63.0 & 64.7 & 1701 & 2965 \\
\hline 1280 & 10 & 65.9 & 68.3 & 1061 & 2133 \\
\hline 1280 & 160 & 66.0 & 68.5 & 1252 & 2195 \\
\hline 1280 & 320 & 66.0 & 68.4 & 1555 & 2966 \\
\hline 1280 & 640 & 65.8 & 68.3 & 1870 & 2948 \\
\hline 1280 & 1280 & 66.0 & 68.4 & 2240 & 2490 \\
\hline 2560 & & 67.6 & 69.1 & 1866 & 2954 \\
\hline 2560 & 160 & 67.8 & 69.3 & 2012 & 2995 \\
\hline 2560 & 320 & 67.8 & 69.4 & 2187 & 2800 \\
\hline 2560 & 640 & 67.6 & 2 & 2370 & 2647 \\
\hline 2560 & 1280 & 67.9 & 69.6 & 2773 & 2535 \\
\hline Proposal met & & 67.7 & 69.3 & 2035 & 1048 \\
\hline
\end{tabular}

Table 5. Recognition accuracy and response time to sequence data

conventional technique, time until an output result is obtained varies very much, the proposed technique has little jitter of response time and outputs a result in very consistent time. In a real-time system, this characteristic is very important when obtaining the prediction possibility of processing.

On the other hand, the proposed technique still has space for improvement. Both accuracy of activity changing point detection and accuracy of recognition in a small window just after an activity changing point are not very high. That is, raising both these accuracies can be expected to shorten response time sharply.

For activity changing point detection, using index Feature Transition Measure instead of STM can raise activity changing point detection accuracy.

\section{CONCLUSION}

This research studied a method to reduce the response time of activity recognition proposed in earlier research using HASC Corpus, which contains large-scale data of people's activities. Furthermore, the suitable

parameters in internal processing were examined. Also, we compared the proposed method with the conventional method and clarified a characteristic of the proposed method.

In the proposed method, first we detected the activity changing point of activity from sensor data. Next, we set up a smaller-than-usual window shortly after detecting the activity changing point. Then we recognized changes using special recognition classifier. Therefore, we could reduce the response time from the activity changing point of activity to obtain result. In this research, we clarify the suitable number of filters and its frequency for the IIR band pass filter bank when detecting activity changing point from a transit moving activity to another activity in activity changing point detection. We clarified the frequency and appropriate number of filters. In addition, we clarified the relationship between identification accuracy and the size of a special window that is set after activity changing point detection.

Comparing the proposed method with the conventional method confirmed that the proposed method reduced the response time to $2035 \mathrm{~ms}$ on average from $2773 \mathrm{~ms}$, the average of the conventional method. The conventional method cannot achieve both high recognition accuracy and short response time. The computational effort of the conventional method is increased if the response time is shortened. On the other hand, we clarified the proposed method can reduce the amount of calculation and achieve both high recognition accuracy and short response time. The proposed method outputs the recognition results in consistent times to reduce the jitter of response time.

\section{References}

[1] Bao, L. and Intille, S. S. : Activity Recognition

fromUser-annotated Acceleration Data, Pervasive 2004, LectureNotes in Computer Science, Vol. 3001

SpringerBerlin / Heidelberg, pp. 117 (2004). 
[2] Cho, I. -Y., Sunwoo, J., Son, Y. -K., Oh, M. -H. and Lee, C. -H. : Development of a Single 3-Axis Accelerometer Sensor Based Wearable Gesture Recognition Band,Ubiquitous Intelligence and Computing, Lecture Notes in Computer Science, Vol. 4611, Springer Berlin / Heidelberg,pp. 43 52, 2007.

[3] Fujimoto, M., Fujita, N., Takegawa, Y. , Terada, T. and Tsukamoto, M. : A Motion Recognition Method for a Wearable Dancing Musical Instrument, Wearable Computers,IEEE International Symposium, pp. 1118 (2009).

[4] Human Activity Sensing Consortium: HASC Challenge 2012, http://hasc. jp/hc2012/index. html. Accesse date: January 142013.

[5] Lester, J., Choudhury, T. and Borriello, G. : A Practical Approach to Recognizing Physical Activities, PERVASIVE 2006 , Lecture Notes in Computer Science, Vol. 3968, Springer Berlin / Heidelberg, pp. 1-16 (2006).

[6] S. Furui : On the role of spectral transition for speech perception, Journal of the Acoustical Society of America,Vol. 80, Issue 4, pp.1016-1025 (1986).

[7] R. Ohmura, N. Hashida, and M. Imai: Exploiting Auto-segmentation Technique for Semi-supervised Learning in Accelerometer-based Activity Recognition, Ubicomp 2010 (2010).

[8] Suutala, J. , Pirttikangas, S. and R"oning, J. : Discriminative Temporal Smoothing for Activity
Recognition from Wearable Sensors, Ubiquitous

Computing Systems 2007 , Lecture Notes in Computer Science, Vol. 4836, Springer Berlin / Heidelberg, pp. 182-195 (2007)

[9] The University of Waikato. :Weka, http://www. cs. waikato. ac. $\mathrm{nz} / \mathrm{ml} /$ weka/. Accesse date: January 14 2013.

[10] R. Ohmura, F. Naya, H. Noma, and K.

koqure :Sensor- Data-Based Nursing Activity Analysis Support System, Transactions of the Virtual Reality Society of Japan, Vol. 14, No. 1, pp. 67-78 (2009).

[11] K. Murao, and T. Terada: A Motion Recognition Method by Constancy Decision, Transactions of Information Processing Society of Japan Vol.52, No. 6, pp. 19681979 (2011).

[12] W. Takasaki and R. Ohmura: Response time reduction of the acceleration base activity recognition by the detecting activity activity changing point information processing society of Japan, 2011-UBI31(2011).

[13] Sozo Inoue, Yuichi Hattori : Toward High-Level ActivityRecognition from Accelerometers on Mobile Phones,Proc. of the 2011 Intl Conference on Internet of Thingsand 4th International Conference on Cyber, Physical and Social Computing, Pp. 225-231 (2011). 